Nervenarzt 2020 $91: 511-517$

https://doi.org/10.1007/s00115-020-00893-1

Online publiziert: 27. März 2020

(c) Der/die Autor(en) 2020

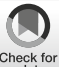

\section{Hans-Christoph Diener' $\cdot$ Matthias Endres ${ }^{2}$}

'Institut für Medizinische Informatik, Biometrie und Epidemiologie (IMIBE), Medizinische Fakultät der Universität Duisburg-Essen, Essen, Deutschland

${ }^{2}$ Klinik und Hochschulambulanz für Neurologie, Charite - Universitätsmedizin Berlin, Berlin, Deutschland

\title{
Vergangenheit und Zukunft des ESUS-Konzepts
}

\section{Hintergrund}

Die Klassifikation ischämischer Schlaganfälle erfolgt traditionell nach den TOAST-Kriterien [1]. Unterschieden werden hier die zerebrale Makroangiopathie mit $>50 \%$ igen Stenosen oder Verschlüssen der extra- oder intrakraniellen Arterien, kardioembolische Schlaganfälle meist im Rahmen von Vorhofflimmern, lakunäre Infarkte im Rahmen einer unbehandelten Hypertonie oder eines schlecht eingestellten Diabetes mellitus und seltene Ursachen wie beispielsweise Vaskulitiden oder Dissektionen. Es verblieb dann eine Untergruppe von Patienten deren zerebrale Insulte als kryptogen eingestuft wurden. Kryptogene Schlaganfälle konnten entweder bedingt sein durch fehlende diagnostische Abklärung, mehrere konkurrierende Mechanismen oder tatsächlich kryptogen, d. h. ungeklärter Ätiologie.

Das Konzept der kryptogenen Schlaganfälle war jedoch nicht ausreichend, um Patienten zu definieren, die potenziell an randomisierten klinischen Therapiestudien teilnehmen können. Aus diesem Grund hat im Jahr 2014 eine internationale Arbeitsgruppe das Konzept des „Embolic Stroke of Undetermined Source“ (ESUS) entwickelt und operationale Kriterien für die Diagnose ESUS publiziert [2].

Die Diagnose ESUS umfasst:

1. Nachweis eines ischämischen Insults in der zerebralen Bildgebung mit CT oder Magnetresonanztomographie und Ausschluss lakunärer Infarkte,

2. Ausschluss einer Makroangiopathie extra- oder intrakranieller hirnver- sorgender Gefäße mit einer über

50\%igen Lumeneinengung,

3. Ausschluss kardial-embolischer Ursachen durch ein 24-StundenEKG und eine transthorakale und transösophageale Echokardiographie,

4. Ausschluss anderer seltener Ursachen wie beispielsweise Vaskulitis, Dissektion, Migräne oder Drogenmissbrauch.

Die zugrunde liegende Hypothese von ESUS war, dass die meisten Schlaganfälle bei diesen Patienten durch embolische Mechanismen bedingt sind [2]. Im Rahmen der Sekundärprävention sollten daher Antikoagulanzien wirksamer sein als eine Behandlung mit Thrombozytenfunktionshemmern.

\section{Epidemiologie}

Epidemiologische Studien zeigten, dass etwa $7-42 \%$ aller Schlaganfälle durch ESUS bedingt sind [3]. Die epidemiologischen Studien mit >1500 Patienten berichten über Prozentsätze von $7 \%$ [4], $9 \%$ [5], $10 \%$ [6] bzw. $16 \%$ [7]. Das mittlere Alter dieser Patienten beträgt 65 Jahre und das jährliche Risiko eines erneuten Schlaganfalls beträgt im Mittel 4,5\%. Die Schlaganfälle im Rahmen von ESUS sind leicht bis mittelschwer und die Mortalität ist gering [3]. Somit ist das Rezidivrisiko nach ESUS durchaus relevant; gleichzeitig zeigt sich aber auch, dass sich ESUSPatienten bez. Erkrankungsalter und Rezidivrisiko deutlich von Patienten mit Vorhofflimmern unterscheiden, die deutlich älter sind und unter ASS oder ohne Behandlung ein jährliches Rezidivrisiko über $10 \%$ aufweisen.

\section{Randomisierte Studien (• Tab. 1)}

Zur Überprüfung der Hypothese, ob orale Nicht-Vitamin-K-Antikoagulanzien (NOAKs) bei Patienten mit ESUS wirksamer sind als Thrombozytenfunktionshemmer wie Acetylsalicylsäure (Aspirin), wurden zwei große randomisierte Studien durchgeführt, nämlich RE-SPECT ESUS (Randomized, DoubleBlind, Evaluation in Secondary Stroke Prevention Comparing the Efficacy and Safety of the Oral Thrombin Inhibitor Dabigatran Etexilate versus Acetylsalicylic Acid in Patients with Embolic Stroke of Undetermined Source; [8, 9]) und NAVIGATE ESUS (New Approach Rivaroxaban Inhibition of Factor Xa in a Global Trial versus ASA to Prevent Embolism in Embolic Stroke of Undetermined Source; $[10,11])$. Die RESPECT-ESUS-Studie war eine Phase3-Studie, die 5390 Patienten mit ESUS einschloss. Die Patienten wurden randomisiert und erhielten entweder 2-mal $150 \mathrm{mg}$ Dabigatran täglich oder bei einem Alter $\geq 75$ Jahre oder einer Kreatininclearance $\leq 50 \mathrm{ml} / \mathrm{min} 2$-mal $110 \mathrm{mg}$ Dabigatran täglich. Die Vergleichsgruppe erhielt $100 \mathrm{mg}$ Acetylsalicylsäure pro Tag. Der mediane Beobachtungszeitraum betrug 19 Monate. Der primäre Wirksamkeitsendpunkt war ein Schlaganfall jedweder Genese und der primäre Sicherheitsendpunkt umfasste schwerwiegende Blutungen.

Die NAVIGATE-ESUS-Studie schloss 7231 Patienten mit ESUS ein und randomisierte diese zu $15 \mathrm{mg}$ Rivaroxaban 1-mal täglich oder $100 \mathrm{mg}$ Acetylsalicylsäure 1-mal täglich. Der primäre Endpunkt war ein Schlaganfall und eine sys- 
Tab. 1 Charakteristika der RE-SPECT-ESUS- und NAVIGATE-ESUS-Studie

\begin{tabular}{|l|l|l}
\hline & RE-SPECT ESUS & NAVIGATE ESUS \\
\hline Zahl der Patienten & 5390 & 7231 \\
\hline NOAK & $\begin{array}{l}\text { Dabigatran } \\
2 \times 150 \mathrm{mg} \\
2 \times 110 \mathrm{mg}\end{array}$ & $\begin{array}{l}\text { Rivaroxaban } \\
1 \times 15 \mathrm{mg}\end{array}$ \\
\hline Alter & 64,5 & \\
\hline Männliches Geschlecht & $63 \%$ & 66,9 \\
\hline Hypertonie & $74 \%$ & $62 \%$ \\
\hline Diabetes mellitus & $22 \%$ & $77 \%$ \\
\hline PFO & $12 \%$ & $25 \%$ \\
\hline Vorheriger Schlaganfall/TIA & $18 \%$ & $7,1 \%$ \\
\hline Mediane Zeit bis zur Randomisierung (Tage) & 46,0 & $17 \%$ \\
\hline Mittlere Beobachtungszeit (Monate) & 19 & 37,0 \\
\hline Intrakranielle Stenosen <50\% & Erlaubt & 11 \\
\hline NOAK Nicht-Vitamin-Korale Antikoagulantien, PFO offenes formane ovale & Ausgeschlossen \\
\hline
\end{tabular}

Tab. 2 Ergebnisse von RE-SPECT ESUS und NAVIGATE ESUS (Ereignisse pro Jahr)

\begin{tabular}{|l|l|l|l|l}
\hline & \multicolumn{2}{l}{ RE-SPECT ESUS } & \multicolumn{2}{l}{ NAVIGATE ESUS } \\
& $\begin{array}{l}\text { Dabigatran } \\
\text { (\%) }\end{array}$ & $\begin{array}{l}\text { Aspirin } \\
(\%)\end{array}$ & $\begin{array}{l}\text { Rivaroxaban } \\
(\%)\end{array}$ & $\begin{array}{l}\text { Aspirin } \\
(\%)\end{array}$ \\
\hline Schlaganfall & 4,1 & 4,8 & 5,1 & 4,7 \\
\hline $\begin{array}{l}\text { Ischämischer Schlaganfall } \\
\text { Zerebrale Blutung }\end{array}$ & 4,0 & 4,7 & 4,7 & 4,7 \\
\hline $\begin{array}{l}\text { Schlaganfall, Myokardinfarkt, vasku- } \\
\text { lärer Tod }\end{array}$ & 0,1 & 0,2 & 0,4 & 0,1 \\
\hline $\begin{array}{l}\text { Schlaganfall mit schwerer Behinde- } \\
\text { rung }\end{array}$ & 0,6 & 5,4 & 6,2 & 5,8 \\
\hline $\begin{array}{l}\text { Mortalität } \\
\text { Schwerwiegende Blutung }\end{array}$ & 1,2 & 0,9 & 1,2 & 0,8 \\
\hline Zerebrale Blutung & 1,7 & 1,3 & 1,9 & 1,5 \\
\hline & 0,1 & 0,2 & 0,3 & 0,7 \\
\hline
\end{tabular}

temische Embolie und der primäre Sicherheitsendpunkt schwerwiegende Blutungskomplikationen nach ISTH-Kriterien.

Die Patienten in den beiden Studien waren im Mittel zwischen 64 und 67 Jahre alt und $62 \%$ waren männlich (• Tab. 1). Hierbei hatten $74-77 \%$ der Patienten eine arterielle Hypertonie und $22-20 \%$ einen Diabetes mellitus. Etwa $18 \%$ der Patienten hatten bereits in der Vorgeschichte einen Schlaganfall oder eine TIA erlitten. Die mediane Zeit vom qualifizierenden Schlaganfall bis zur Randomisierung betrug 38 Tage bei NAVIGATE ESUS und 46 Tage bei RE-SPECT ESUS.

\section{Ergebnisse (• Tab. 2)}

In der RE-SPECT-ESUS-Studie traten in der Behandlungsgruppe mit Dabigatran 177 Ereignisse auf. Dies entspricht 4,1 \%/ Jahr. In der Gruppe mit Acetylsalicylsäure kam es zu 207 erneuten Schlaganfällen, entsprechend 4,8\%/Jahr. Die daraus resultierende Hazard Ratio betrug 0,85 mit einem $95 \%$-Konfidenzintervall von $0,69-1,03$ und ein $p$-Wert von 0,10 . Im Rahmen einer Landmark-Analyse wurde die Studie im ersten und im darauffolgenden Jahr ausgewertet. Für das erste Jahr der Beobachtungszeit betrug die schen der Dabigatran-Gruppe und den Patienten, die Acetylsalicylsäure erhielten, 0,99 mit einem 95\%-Konfidenzintervall von $0,79-1,25$. Für den Zeitraum nach einem Jahr betrug die Hazard Ratio Hazard Ratio für den Therapieeffekt zwi-
0,50 zugunsten der Dabigatran-Gruppe mit einem $95 \%$-Konfidenzintervall von $0,32-0,77$.

Die NAVIGATE-ESUS-Studie wurde nach einer medianen Beobachtungszeit von 11 Monaten vorzeitig abgebrochen. Der primäre Endpunkt der Studie aus Schlaganfall und systemischer Embolie trat bei 172 Patient in der RivaroxabanGruppe auf, entsprechend einer jährlichen Rate von $5,1 \%$, und bei 160 Patienten in der Aspirin-Gruppe, entsprechend einer jährlichen Rate von $4,7 \%$. Dieser Unterschied entspricht einer Hazard Ratio von 1,07 mit einem 95\%-Konfidenzintervall von $0,87-1,33$ und ein $p$-Wert von 0,52 .

Beide Studien zeigen im ersten Jahr keinen Unterschied zwischen der Antikoagulation und Aspirin. In der RE-SPECTESUS-Studie beginnen die Kaplan-Meier-Kurven für erneute Schlaganfälle zu divergieren mit einem Vorteil für Dabigatran. NAVIGATE ESUS wurde nach einer mittleren Beobachtungszeit von 11 Monaten abgebrochen, sodass unklar bleibt, ob ein ähnliches Phänomen wie in RESPECT ESUS zu beobachten gewesen wäre. Interessanterweise zeigte sich in der COMPASS-Studie (Cardiovascular Outcomes for People Using Anticoagulation Strategies) ein ähnliches Phänomen, auch hier war der Vorteil von NOAK gegenüber ASS erst nach dem ersten Jahr zu beobachten [23].

Beide Studien berichteten auch über wichtige sekundäre Endpunkte. In der RE-SPECT-ESUS-Studie betrug die Häufigkeit erneuter ischämischer Schlaganfälle $172(4 \% / \mathrm{Jahr})$ in der DabigatranGruppe und 203 (4,7\%) in der AspirinGruppe. Dies entspricht einer Hazard Ratio von 0,84 . Die Sterblichkeit war nicht unterschiedlich und betrug 56 Patienten $(1,2 \% / J a h r)$ in der Dabigatran-Gruppe und 58 Patienten ( $1,3 \% / \mathrm{Jahr}$ ) in der Aspirin-Gruppe mit einer Hazard Ratio von 0,96 .

In der NAVIGATE-ESUS-Studie kam es zu 158 ischämischen Schlaganfällen (4,7\%/Jahr) in der Rivaroxaban-Gruppe und zu 156 ischämischen Schlaganfällen in der Aspirin-Gruppe (4,7\%/Jahr) mit einer Hazard Ratio von 1,01. Die Gesamtsterblichkeit betrug 65 Patienten in der Rivaroxaban-Gruppe (1,9\%/Jahr) 
und 52 Patienten in der Aspirin-Gruppe (1,5\%/Jahr) mit einer Hazard Ratio von 1,26 .

Schwerwiegende Blutungskomplikation traten in der RE-SPECT-ESUSStudie bei 77 Patienten in der Dabigatran-Gruppe auf, entsprechend 1,7\%/ Jahr, und bei 64 Patienten (1,4\%/Jahr) in der Aspirin-Gruppe mit einer Hazard Ratio von 1,19 und einem 95\%-Konfidenzintervall von 0,85-1,66 und einem p-Wert von 0,30 . In der NAVIGATEESUS-Studie kam es bei 62 Patienten der Rivaroxaban-Gruppe, entsprechend $1,8 \% / \mathrm{Jahr}$, und bei 23 Patienten in der Aspirin-Gruppe, entsprechend 0,7\%/ Jahr, zu einer schwerwiegenden Blutungskomplikation. Dies resultierte in einer Hazard Ratio von 2,72 und einem $95 \%$-Konfidenzintervall von $1,68-4,39$ und einem $p$-Wert von $<0,001$.

Vergleicht man die Blutungskomplikation in den beiden Studien sind die jährlichen Blutungsraten für die Rivaroxaban- $(1,8 \% / \mathrm{Jahr})$ und die Dabigatran-Gruppe (1,7\% Jahr) ähnlich. Es ergeben sich deutliche Unterschiede in den Patientengruppen, die mit Aspirin behandelt wurden. So betrug die jährliche Rate schwerwiegende Blutungen für Aspirin in der NAVIGATE EUS Studie $0,7 \% /$ Jahr und in der RE-SPECT-ESUSStudie 1,4\%/Jahr. Diese Unterschiede sind schwierig $\mathrm{zu}$ erklären, da beide Studien identische Baseline-Parameter hatten und die Population daher bezüglich ihres Blutungsrisikos vergleichbar waren. NAVIGATE ESUS verwendete eine mikroverkapselte Form von Aspirin, die möglicherweise zu einer Reduktion der Rate schwerwiegender Blutungen geführt hat. Mikroverkapseltes Aspirin reduziert das Risiko gastrointestinaler Blutungen. Leider wurde die Häufigkeit gastrointestinaler Blutungen in NAVIGATE ESUS nicht publiziert. Der größte Unterschied zeigte sich bei den intrakraniellen Blutungen. Diese betrugen $0,1 \% / \mathrm{Jahr}$ in der NAVIGATE-ESUSStudie und 0,7\%/Jahr in der RE-SPECTESUS-Studie. Hier kann aber eigentlich die Tatsache, dass in der NAVIGATEESUS-Studie Aspirin mikroverkapselt war, keine Rolle spielen, da dies nur Auswirkungen auf gastrointestinale Blutungen hätte.

Nervenarzt 2020 $91: 511-517$ https://doi.org/10.1007/s00115-020-00893-1

(c) Der/die Autor(en) 2020

\section{H.-C. Diener $\cdot$ M. Endres}

\section{Vergangenheit und Zukunft des ESUS-Konzepts}

\section{Zusammenfassung}

Schlaganfälle werden traditionell nach den TOAST-Kriterien eingeteilt. Das Konzept des kryptogenen Schlaganfalls erfüllte allerdings nicht die operationalen Kriterien, die notwendig waren, um Patientenpopulation zu definieren, die für randomisierte Studien im Betracht kamen. Daher wurde das Konzept des Embolic Stroke of Undetermined Etiology (ESUS) entwickelt. Eine zugrunde liegende Hypothese war, dass die meisten Schlaganfälle bei Patienten mit ESUS durch embolische Ereignisse bedingt sind und weitere Ereignisse durch eine Antikoagulation verhindert werden können.
Deshalb wurden zwei große randomisierte Studien durchgeführt, die Dabigatran oder Rivaroxaban mit Acetylsalicylsäure verglichen. Beide Studien konnten keine Überlegenheit des NOAK im Vergleich zu Aspirin zeigen. Subgruppenanalysen zeigen allerdings, dass es Patientenpopulation gibt, die möglicherweise doch von einer oralen Antikoagulation profitieren.

Schlüsselwörter

Schlaganfall - Embolischer Schlaganfall ungeklärter Ätiologie · Antikoagulation . Aspirin · Blutungskomplikationen

\section{Past and future of the ESUS concept}

\section{Abstract}

Stroke has traditionally been classified according to the trial of ORG 10172 in acute stroke treatment (TOAST) criteria; however, the concept of cryptogenic stroke did not meet the operational criteria necessary to define patient populations eligible for randomized studies. Therefore, the concept of embolic stroke of undetermined etiology (ESUS) was developed. An underlying hypothesis was that most strokes in patients with ESUS are caused by embolic events, hence, anticoagulation may prevent secondary events. Therefore, two large randomized trials were conducted comparing dabigatran or rivaroxaban with acetylsalicylic acid. Both studies could not show superiority of the new oral anticoagulants (NOAC) compared to aspirin; however, subgroup analyses showed that there is a patient population that may benefit from oral anticoagulation.

\section{Keywords}

Stroke Embolic stroke of undetermined source $\cdot$ Anticoagulation - Aspirin · Bleeding complications

\section{Subgruppenanalysen (๑ Tab. 3)}

\section{Offenes Foramen ovale}

In beiden Studien wurden Patienten mit offenem Foramen ovale (PFO) durch Echokardiographie identifiziert. In der NAVIGATE-ESUS-Studie betrug die Identifikationsrate eines offenen Foramen ovale $7,4 \%$ der Patienten [12]. Dies betraf 4,6\% der 6884 Patienten, die eine transthorakale Echokardiographie erhielten, und 27,4\% der 1082 Patienten, die eine transösophageale Echokardiographie erhielten. Einen erneuten ischämischen Schlaganfall erlitten 275 Patienten, die Aspirin erhielten, und 259 Patienten, die mit Rivaroxaban behandelt wurden. Dies entspricht einer Hazard Ratio von 0,54 und einem $95 \%$ -
Konfidenzintervall von $0,22-1,36$. Die Studiengruppe führte dann eine Metaanalyse durch und verglich eine orale Antikoagulation mit Acetylsalicylsäure bei Patienten mit offenem Foramen ovale und ESUS in der PICSS-Studie [13], in der CLOSE-Studie [14] sowie in NAVIGATE ESUS. Die Metaanalyse ergab eine Hazard Ratio zugunsten der Antikoagulation von 0,54 mit einem $95 \%$-Konfidenzintervall von $0,24-0,96$. In der RE-SPECT-ESUS-Studie konnte jedoch ein möglicher Vorteil der Antikoagulation in der PFO-Subgruppe nicht bestätigt werden: Hier betrug die Hazard Ratio zwischen der DabigatranGruppe und der Aspirin-Gruppe 0,83 bei den Patienten, die kein offenes Foramen ovale hatten, und 0,88 bei den Patienten, die ein offenes Foramen ovale 
Tab. 3 Ergebnisse von Subgruppenanalysen der NAVIGATE-ESUS- und RE-SPECT-ESUS-Studie

\begin{tabular}{|c|c|c|}
\hline Untergruppe & RE-SPECT ESUS & NAVIGATE ESUS \\
\hline $\begin{array}{l}\text { Offenes Foramen } \\
\text { ovale }\end{array}$ & Dabi $=$ ASA & Riva $>$ ASA (Trend) \\
\hline Disabling Stroke & Dabi $>$ ASA & Riva $=$ ASA \\
\hline Alter & $>75$ Jahre Dabi > ASA & $<60$ Jahre ASA > Riva \\
\hline Nierenfunktion & $\begin{array}{l}\mathrm{CrCl}<50 \mathrm{ml} / \mathrm{min} \mathrm{Da}- \\
\mathrm{bi}>\mathrm{ASA}\end{array}$ & $\mathrm{CrCl}<50 \mathrm{ml} / \mathrm{min}$ Riva $=\mathrm{ASA}$ \\
\hline $\begin{array}{l}\text { Prädiktoren für Vor- } \\
\text { hofflimmern }\end{array}$ & $\begin{array}{l}\text { Alter, Hypertonie, Nieren- } \\
\text { erkrankung }\end{array}$ & $\begin{array}{l}\text { Früherer Schlaganfall/TIA, Nikotinkonsum, } \\
\text { Alter, Diabetes mellitus, multiple Infarkte }\end{array}$ \\
\hline $\begin{array}{l}\text { Vorhof und Schlagan- } \\
\text { fallrisiko }\end{array}$ & Nicht untersucht & $\begin{array}{l}\text { Bei Durchmesser linker Vorhof }>4,6 \mathrm{~cm} \\
\text { Riva }>\text { ASA }\end{array}$ \\
\hline $\begin{array}{l}\text { Bestehende Arterio- } \\
\text { sklerose }\end{array}$ & Nicht untersucht & Riva $=$ ASA \\
\hline Dabi Dabigatran, ASA & 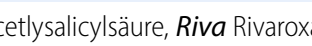 & an, $\mathrm{CrCl}$ Kreatininclearance \\
\hline
\end{tabular}

aufwiesen. Wurde dann die Metaanalyse mit den Ergebnissen von RE-SPECT ESUS aktualisiert, traten 28 Ereignisse bei den Patienten auf, die antikoaguliert waren, und 45 bei den Patienten auf, die Aspirin erhielten. Dies entspricht einer Hazard Ratio von 0,70 mit einem $95 \%$-Konfidenzintervall von 0,43-1,14. Der Unterschied war somit nicht mehr signifikant.

\section{Schwerwiegende Schlaganfälle}

Beide Studien analysierten erneute Schlaganfälle mit der modifizierten Rankin-Skala. In RE-SPECT ESUS zeigte sich eine Rate schwerwiegender Schlaganfälle oder von Tod für die DabigatranGruppe von $0,6 \% / \mathrm{Jahr}$ verglichen mit Aspirin mit 0,9\%/Jahr. Die entsprechenden absoluten Zahlen betrugen 25 und 42 Patienten und die daraus resultierende Hazard Ratio von 0,519 war mit einem $95 \%$-Konfidenzintervall von 0,36-0,96 signifikant (• Tab. 2). In der NAVIGATE-ESUS-Studie ergaben sich keine signifikanten Unterschiede für schwerwiegende Schlaganfälle und Tod zwischen Rivaroxaban und Aspirin.

\section{Alter}

In der NAVIGATE-ESUS-Studie ergaben sich Unterschiede zwischen den beiden Therapieansätzen in Abhängigkeit vom Alter. Bei <60-jährigen Patienten traten 43 Schlaganfälle in der Rivaroxaban und 25 in der Aspirin-Gruppe auf. Dies entspricht einer signifikanten Haz- ard Ratio zugunsten von Aspirin von 1,73 mit einem 95\%-Konfidenzintervall von 1,06-2,83. Für Patienten im Alter $>60$ Jahre ergaben sich keine signifikanten Unterschiede zwischen Rivaroxaban und Aspirin (HR 0,94-0,97). In der RESPECT-ESUS-Studie ergab sich eine signifikante Überlegenheit von Dabigatran im Vergleich zur Aspirin für Patienten im Alter $\geq 75$ Jahre. Hier traten 43 Schlaganfälle $(7,8 \% / J a h r)$ in der DabigatranGruppe auf und 60 (12,4\%/Jahr) in der Aspirin-Gruppe. Dies führte zu einer Hazard Ratio von 0,63 (Konfidenzintervall $0,43-0,94)$ und einem $p$-Wert von 0,023 . Für Patienten $<75$ Jahre bestanden keine Unterschiede zwischen Dabigatran und Aspirin. Diese Beobachtung würde die Hypothese stützen, dass bei älteren Patienten im Verlauf ein höheres Risiko besteht, stummes Vorhofflimmern zu entwickeln und dann im Verlauf von einer Antikoagulation zu profitieren.

\section{Dosierung von Dabigatran}

Bei Patienten $\geq 75$ Jahre oder einer Kreatininclearance $\leq 50 \mathrm{ml} / \mathrm{min}$ wurde die Dosis von Dabigatran auf 2-mal 110 mg erniedrigt. Es ergaben sich signifikante Unterschiede zwischen der Dabigatran- und Aspirin-Gruppe in Abhängigkeit von der verwendeten Dabigatran-Dosis. Für die 2-mal 150-mg-Dosis ergaben sich keine Unterschiede für erneute Schlaganfälle zwischen Dabigatran und Aspirin (Hazard Ratio 1,0). Für die 2-mal tägliche Gabe von $110 \mathrm{mg}$ Dabigatran ergaben sich 45 Ereignisse in der Dabigatran-Gruppe
(7,4\%/Jahr) verglichen mit 71 Ereignissen unter Aspirin (13\%/Jahr). Dies resultierte in einer signifikanten Hazard Ratio von $0,57(0,39-0,82)$ zugunsten von Dabigatran und einem $p$-Wert von 0,03 . Dieses Ergebnis war zu erwarten, da der Grund für die Dosisreduktion bei den meisten Patienten das Alter war.

\section{Nierenfunktion}

In der RE-SPECT-ESUS-Studie ergaben sich keine Unterschiede bei Patienten mit einer normalen Nierenfunktion gemessen als eine Kreatininclearance von $\geq 50 \mathrm{ml} / \mathrm{min}$. Für Patienten mit einer Kreatininclearance zwischen 30 und $50 \mathrm{ml} / \mathrm{min}$ und einer DabigatranDosis von 2-mal $110 \mathrm{mg}$ ergaben sich 23 Ereignisse bei Dabigatran und 33 in der Aspirin-Gruppe. Dies führte zu einer nichtsignifikanten Reduktion von Schlaganfällen und einer Hazard Ratio von 0,63 mit einem $p$-Wert von 0,087 . In der NAVIGATE-ESUS-Studie zeigten Patienten mit einer Kreatininclearance zwischen 30 und $50 \mathrm{ml} / \mathrm{min}$ keinen Unterschied zwischen Rivaroxaban und Aspirin für den Endpunkt Schlaganfall und systemische Embolie. Bei Patienten mit einer Kreatininclearance über $80 \mathrm{ml} / \mathrm{min}$ ergab sich eine Hazard $\mathrm{Ra}$ tio von 1,57 zugunsten von Aspirin mit einem $95 \%$-Konfidenzintervall von $1,11-2,23$.

\section{EKG-Monitoring}

In der RE-SPECT-ESUS-Studie war ein Langzeit-EKG-Monitoring zulässig. Von 2695 Patienten in der Dabigatran-Gruppe wurde bei 208 Patienten, entsprechend $7,7 \%$, im Verlauf der Studie Vorhofflimmern entdeckt. Die entsprechende Zahl bei den 2695 Patienten unter Aspirin betrug 195. Die mediane Zeit von der Randomisierung bis zur Identifikation von Vorhofflimmern betrug 232 Tage in der Dabigatran-Gruppe und 96 Tage in der Aspirin-Gruppe. Die Rate erneuter Schlaganfälle nach der Identifikation von Vorhofflimmern und Umstellung auforale Antikoagulation betrug 5,7\%/Jahr bei den Patienten, die ursprünglich mit $\mathrm{Da}$ bigatran behandelt worden waren, und 
10,5\%/Jahr bei den Patienten, die mit Aspirin behandelt worden waren.

\section{Prädiktoren für Vorhofflimmern}

In der RE-SPECT-ESUS-Studie wurden Prädiktoren für das Auftreten von Vorhofflimmern identifiziert. Die Berechnung erfolgte über eine multivariable Regressionsanalyse. Signifikante Prädiktoren für das Auftreten von Vorhofflimmern waren Alter (kleiner vs. größer 75 Jahre), das Vorliegen einer Hypertonie und eine Anamnese mit einer Nierenerkrankung. Nicht signifikant waren koronare Herzerkrankung, früherer Schlaganfall oder TIA, eine Kreatininclearance $\leq 50 \mathrm{ml} / \mathrm{min}$ und ein Diabetes mellitus. Bei den Laborparametern war NT-proBNP ein signifikanter Prädiktor für das Auftreten von Vorhofflimmern.

In der NAVIGATE-Studie wurden sieben Prädiktoren für erneute Schlaganfälle gefunden [15]: Schlaganfall oder TIA vor dem qualifizierenden Schlaganfall (Hazard Ratio 2,03; $95 \%$-CI 1,58-2,60), aktueller Tabakkonsum (HR 1,62, $95 \%$ CI 1,24-2,12), Alter (HR 1,02 pro Jahr Zunahme, 95\%-CI 1,01-1,03), Diabetes mellitus (HR 1,28, $95 \%$-CI 1,01-1,64), multiple akute Infarkte in der zerebralen Bildgebung (HR 1,49, 95\%-CI 1,09-2,02), Einnahme von Aspirin vor dem qualifizierten Schlaganfall (HR 1,34, $95 \%$-CI 1,02-1,70) und Zeit vom qualifizierten Schlaganfall bis zur Randomisierung (HR 0,98, 95\%-CI 0,97-0,99). Die Rate der rezidivierenden Schlaganfälle betrug 2,6\% pro Jahr bei Teilnehmern ohne diese Risikofaktoren und stieg um durchschnittlich $45 \%$ für jeden unabhängigen Prädiktor an ( $p<0,001 ;[15])$.

\section{Einfluss der Morphologie des linken Vorhofs auf das Schlaganfallrisiko}

In der NAVIGATE-ESUS-Studie wurde bei 4022 Teilnehmen der Durchmesser des linken Vorhofs in der Echokardiographie gemessen [16]. Der mittlere Durchmesser betrug $3,8 \mathrm{~cm}$. Patienten mit einem Durchmesser von $>4,6 \mathrm{~cm}$ (9\% der Teilnehmer) hatten einen signifikanten Nutzen von Rivaroxaban mit einer jährlichen Schlaganfallrate von 1,7\% vergli- chen mit Aspirin von 6,5\% und einer Hazard Ratio von 0,26 mit einem 95\%Konfidenzintervall von 0,07-0,94.

\section{Einfluss der Arteriosklerose}

Die Studie untersuchte auch, welchen Einfluss atherosklerotische Plaques oder $<50 \%$ ige Stenosen der Karotiden auf die Rate erneuter Schlaganfälle und systemischer Embolien hatten [17]. Sowohl beim Vorliegen atherosklerotischer Plaques wie von $<50 \%$ ige Stenosen ergaben sich keine Unterschiede zwischen Rivaroxaban und Aspirin. In der NAVIGATEESUS-Studie hatten 1382 Teilnehmer eine transösophageale Echokardiographie und 397 (29\%) eine Aortenbogenatherosklerose, davon 112 (8\%) eine komplexe Aortenbogenatherosklerose [18]. Die jährlichen Raten eines ischämischen Schlaganfallrezidivs betrugen $7,2 \%$ gegenüber $4,2 \%$ und gegenüber $5,6 \%$ für komplexe vs. nichtkomplexe vs. keine Aortenbogenatherosklerose. Bei Patienten mit komplexer Aortenbogenatherosklerose traten unter den mit Rivaroxaban und Aspirin behandelten Patienten jeweils 4 Schlaganfälle auf.

\section{Prädiktoren für einen Schlaganfall bei Patienten mit ESUS aus anderen Studien}

Eine Reihe von Studien untersuchte EKG- und echokardiographische Parameter, um Schlaganfälle bei Patienten mit ESUS vorauszusagen. Als signifikante Prädiktoren erwiesen sich dabei im EKG „P-wave terminal force“ in V1 $>5000 \mu \mathrm{V}$ oder eine Vergrößerung des linken Vorhofs [19]. Der Nachweis einer Fibrose des linken Vorhofs im Gadolinium-MRT des Herzens war ebenfalls ein Prädiktor [20]. Keine prädiktiven Eigenschaften hatte eine reduzierte linksventrikuläre Auswurffraktion oder eine reduzierte Bewegung des linken Ventrikels [21]. Eine generelle Vergrößerung des linken Vorhofs korrelierte nicht mit dem Risiko eines erneuten Schlaganfalls bei ESUS, aber mit dem Auftreten von Vorhofflimmern [22].

\section{Kombinationstherapie}

In der COMPASS-Studie, die Patienten mit koronarer Herzerkrankung oder peripher-arterieller Verschlusskrankheit einschloss [23] gab es eine Untergruppe von Patienten, die einen Schlaganfall erlitten [24]. Die Patienten wurden dahingehend analysiert, welche Ätiologie des Schlaganfalls nach den TOASTKriterien vorlag. Bei den Patienten, die einen nachgewiesenen kardioembolischen Infarkt erlitten hatten, war die Kombination von 2,5 mg Rivaroxaban 2-mal täglich plus Aspirin signifikant wirksamer als eine Aspirin-Monotherapie. Dasselbe galt bei den Patienten mit ESUS.

\section{Derzeit laufende Studien}

Zurzeit laufen noch zwei große randomisierte Studien, bei denen Apixaban mit Aspirin bei Patienten mit ESUS verglichen wird. Die ATTICUS-Studie (Apixaban for the treatment of embolic stroke of undetermined source) ist eine multizentrische nationale (Deutschland) randomisierte Studie, die die Überlegenheit von Apixaban im Vergleich zur Aspirin für die Reduktion neuer ischämischer Läsionen in der Magnetresonanztomographie belegen soll [25]. Die geplante Studiengröße umfasst 500 Patienten mit ESUS. Bei allen Patienten erfolgt initial eine Magnetresonanztomographie, die nach 12 und 24 Monaten wiederholt wird. In den wiederholten MRTs sollen dann neue klinisch apparente und klinisch stumme zerebrale Infarkte verglichen werden.

Die ARCADIA-Studie (The AtRial Cardiopathy and Antithrombotic Drugs In prevention After cryptogenic stroke randomized trial) ist eine multizentrische, randomisierte, doppelblinde Studie in den Vereinigten Staaten bei Patienten mit ESUS und einer Kardiopathie des linken Vorhofs. Vorhofkardiopathie wurde definiert als $\geq 1$ der folgenden Marker: P-Wellen-Terminalforce $>5000 \mu \mathrm{V} \times \mathrm{ms}$ in der EKG-Ableitung V1, Serum NTproBNP $>250 \mathrm{pg} / \mathrm{ml}$ und Index des linken Vorhofdurchmessers $\geq 3 \mathrm{~cm} / \mathrm{m}^{2}$ im Echokardiogramm [26]. Verglichen werden Apixaban $5 \mathrm{mg}$ 2-mal täglich mit $81 \mathrm{mg}$ Aspirin 1-mal täglich. 


\section{Schlussfolgerungen}

Im Moment gibt es zwei randomisierte Studien, die keine Überlegenheit eines NOAKs gegenüber Aspirin bei der Sekundärprävention von Patienten mit ESUS gezeigt haben. Zwei Studien werden derzeit noch durchgeführt.

Zum gegenwärtigen Zeitpunkt gibt es somit keine Indikation, Patienten nach ESUS zu antikoagulieren. Grundlage für die Antikoagulation ist somit weiterhin der Nachweis eines Vorhofflimmerns. Bis die Ergebnisse der noch laufenden Studien vorliegen, sollte daher bei ausgewählten Patienten mit hohem Rezidivrisiko ein Langzeit-EKG-Monitoring stattfinden. Dies kann entweder durch ein 10-tägiges Monitoring unmittelbar nach dem Schlaganfall erfolgen sowie nach 3 und 6 Monaten [27]. In Kürze werden auch die Ergebnisse der MONDAFIS-Studie erwartet, die den Nutzen einer systematischen EKG-Diagnostik während des Stroke-Unit-Aufenthaltes hinsichtlich VHF-Detektion, längerfristiger Therapie sowie vaskulären Rezidiven nach 12 Monaten untersucht [28]. Eine weitere Alternative ist die Implantation eines Event-Recorders. Die beiden ESUS-Studie haben jedoch auch gezeigt, dass die „Dunkelziffer“ von bislang nichtentdecktem paroxysmalem VHF bei ESUS-Patienten geringer ist als zuvor angenommen.

Zusammenfassend ist es derzeit nicht sinnvoll, das ESUS-Konstrukt in der bisherigen Definition in eine erweiterte TOAST-Klassifikation aufzunehmen. Die Ergebnisse der verschiedenen Subgruppenanalysen lassen jedoch den Schluss zu, dass eine differenziertere Einteilung pathophysiologisch sinnvoll und auch behandlungsrelevant sein könnte. Als erste Konsequenz sollten Patient mit offenem Foramen ovale nicht mehr in die Kategorie ESUS aufgenommen werden. Vier große randomisierte Studien haben bei Patienten mit ESUS im Alter unter 60 Jahren gezeigt, dass ein interventioneller Verschluss des offenen Foramen ovale einer Therapie mit Thrombozytenfunktionshemmern überlegen ist [29]. Ob eine Antikoagulation ASS bei PFO-Patienten überlegen ist, müsste nochmals gesondert untersucht werden. Patienten mit atherosklerotischen Plaques und Karotisstenosen $<50 \%$ könnten wahrscheinlich von einer modifizierten Therapie mit niedrig dosierten NOAKs in Kombination mit Aspirin profitieren [23].

Besonders interessant ist die Subgruppe der Patienten mit einer atrialen Kardiopathie, die ja in den noch laufenden ESUS-Studien explizit untersucht werden. Denkbar wäre also in Zukunft ein modifiziertes ESUS-Konzept mit Hinzunahme solcher Vorhofparameter.

\section{Fazit für die Praxis}

\section{- ESUS definiert eine Subgruppe von Patienten mit kryptogenem Hirninfarkt, die einen embolischen Schlaganfall erlitten haben. \\ - Zwei große randomisierte Studien zeigen keinen Nutzen einer Antiko- agulation bei Patienten mit ESUS. Diese Patienten sollten weiterhin mit Thrombozytenfunktionshemmern behandelt werden. \\ - Zukünftig könnte möglicherweise ein revidiertes ESUS-Konzept weitere Parameter wie z. B. eine Vorhofkar- diopathie aufnehmen. Dieses wird derzeit in Studien untersucht.}

\section{Korrespondenzadresse}

Prof. Dr. Hans-Christoph Diener Institut für Medizinische Informatik, Biometrie und Epidemiologie (IMIBE), Medizinische Fakultät der Universität Duisburg-Essen Hufelandstraße 55, 45147 Essen, Deutschland hans.diener@uk-essen.de

Funding. Open Access funding provided by Projekt DEAL.

\section{Einhaltung ethischer Richtlinien}

Interessenkonflikt. H.-C. Diener war der Principal Investigator der RE-SPECT-ESUS-Studie. Er hat in den letzten 3 Jahren Honorare für die Teilnahme an klinischen Studien, Mitarbeit in Advisory Boards und Vorträge erhalten von: Abbott, Bristol-Meyers Squibb Boehringer Ingelheim, Daiichi-Sankyo, Medtronic, Novartis, Pfizer, Portola, Sanofi-Aventis und Servier. Er hat Forschungsmittel von folgenden Institutionen erhalten: DFG, BMBF, EU, NIH, EAST-AFnet, Bertelsmann Stiftung und Heinz-Nixdorf Stiftung. H.-C. Diener besitzt keine Aktien oder Anteile von Pharmafirmen oder Medizintechnikfirmen. H.-C. Diener war beteiligt an der Erstellung von Leitlinien der DGN, der DSG, der
ESC und EHRA. M. Endres war der Leiter der Klinischen Prüfung für NAVIGATE ESUS in Deutschland. Er erhielt Unterstützung für Forschungsprojekte von Bayer, Bundesministerium für Bildung und Forschung, Corona Foundation, Deutsche Forschungsgemeinschaft, Deutsches Zentrum für Herz-Kreislauf-Forschung, Deutsches Zentrum für Neurodegenerative Erkrankungen, EU und Foundation Leducq. Honorare wurden an die Charité bezahlt von Boehringer Ingelheim, Bristol-Myers Squibb/Pfizer, Daiichi Sankyo, Amgen, GlaxoSmithKline, Sanofi, Covidien, und Novartis.

Für diesen Beitrag wurden von den Autoren keine Studien an Menschen oder Tieren durchgeführt. Für die aufgeführten Studien gelten die jeweils dort angegebenen ethischen Richtlinien.

Open Access. Dieser Artikel wird unter der Creative Commons Namensnennung 4.0 International Lizenz veröffentlicht, welche die Nutzung, Vervielfältigung Bearbeitung, Verbreitung und Wiedergabe in jeglichem Medium und Format erlaubt, sofern Sie den/die ursprünglichen Autor(en) und die Quelle ordnungsgemäß nennen, einen Link zur Creative Commons Lizenz beifügen und angeben, ob Änderungen vorgenommen wurden.

Die in diesem Artikel enthaltenen Bilder und sonstiges Drittmaterial unterliegen ebenfalls der genannten Creative Commons Lizenz, sofern sich aus der Abbildungslegende nichts anderes ergibt. Sofern das betreffende Material nicht unter der genannten Creative Commons Lizenz steht und die betreffende Handlung nicht nach gesetzlichen Vorschriften erlaubt ist, ist für die oben aufgeführten Weiterverwendungen des Materials die Einwilligung des jeweiligen Rechteinhabers einzuholen.

Weitere Details zur Lizenz entnehmen Sie bitte der Lizenzinformation auf http://creativecommons.org/ licenses/by/4.0/deed.de.

\section{Literatur}

1. Adams HP Jr., Bendixen BH, Kappelle LJ, Biller J, Love BB, Gordon DL et al (1993) Classification of subtype of acute ischemic stroke. Definitions for use in a multicenter clinical trial. TOAST. Tria of Org 10172 in acute stroke treatment. Stroke 24(1):35-41

2. Hart RG, Diener HC, Coutts SB, Easton JD, Granger CB, O'Donnell MJ et al (2014) Embolic strokes of undetermined source: the case for a new clinical construct. Lancet Neurol 13(4):429-438

3. HartRG, Catanese L,Perera KS, NtaiosG, ConnollySJ (2017) Embolic stroke of undetermined source: a systematic review and clinical update. Stroke 48(4):867-872

4. Li L, Yiin GS, Geraghty OC, Schulz UG, Kuker W, Mehta $Z$ et al (2015) Incidence, outcome, risk factors, and long-term prognosis of cryptogenic transient ischaemic attack and ischaemic stroke: a population-based study. Lancet Neurol 14(9):903-913

5. Arauz A, Morelos E, Colin J, Roldan J, Barboza MA (2016) Comparison of functional outcome and stroke recurrence in patients with embolic stroke of undetermined source (ESUS) vs. cardioembolic stroke patients. PLoSONE 11(11):e166091

6. Ntaios G, Papavasileiou V, Milionis H, Makaritsis K, Manios E, Spengos Ket al (2015) Embolic strokes of undetermined source in the athens stroke registry: a descriptive analysis. Stroke 46(1):176-181 
7. Perera KS, Vanassche T, Bosch J, Giruparajah M, Swaminathan B, Mattina KR et al (2016) Embolic strokes of undetermined source: prevalence and patient features in the ESUS global registry. Int J Stroke 11(5):526-533

8. Diener HC, Easton JD, Granger CB, Cronin L, DuffyC, Cotton D et al (2015) Design of randomized, double-blind, evaluation in secondary stroke prevention comparing the EfficaCy and safety of the oral thrombin inhibitor dabigatran etexilate vs. acetylsalicylic acid in patients with embolic stroke of undetermined source (RE-SPECT ESUS). Int J Stroke 10(8):1309-1312

9. Diener $H C$, Sacco RL, Easton JD, Granger $C B$, Bernstein RA, Uchiyama S et al (2019) Dabigatran for prevention of stroke after embolic stroke of undetermined source. $\mathrm{N}$ Engl J Med 380(20):1906-1917

10. Hart RG, Sharma M, Mundl H, Shoamanesh A, Kasner SE, Berkowitz SD et al (2016) Rivaroxaban for secondary stroke prevention in patients with embolic strokes of undetermined source: design of the NAVIGATE ESUS randomized trial. Eur Stroke J 1(3):146-154

11. Hart RG, Sharma M, Mundl H, Kasner SE, Bangdiwala SI, Berkowitz SD et al (2018) Rivaroxaban for stroke prevention after embolic stroke of undetermined source. N Engl J Med 378(23):2191-2201

12. Kasner SE, Swaminathan B, Lavados P, Sharma M, Muir K, Veltkamp R et al (2018) Rivaroxaban or aspirin for patent foramen ovale and embolic stroke of undetermined source: a prespecified subgroup analysis from the NAVIGATE ESUS trial. Lancet Neurol 17(12):1053-1060. https://doi.org/ 10.1016/S1474-4422(18)30319-3

13. Homma S, Sacco RL, Di Tullio MR, Sciacca RR, Mohr JP (2002) Effect of medical treatment in stroke patients with patent foramen ovale: patent foramen ovale in cryptogenic stroke study. Circulation 105(22):2625-2631

14. Mas JL, Derumeaux G, Guillon B, Massardier E, Hosseini H, MechtouffLetal (2017) Patentforamen ovale closure or anticoagulation vs. antiplatelets after stroke. NEngl J Med 377(11):1011-1021

15. Hart RG, Veltkamp RC, Sheridan P, Sharma M, Kasner SE, Bangdiwala SI et al (2019) Predictors of recurrent ischemic stroke in patients with embolic strokes of undetermined source and effects of rivaroxaban versus aspirin according to risk status: the NAVIGATE ESUS trial. J Stroke Cerebrovasc Dis 28(8):2273-2279

16. Healey JS, GladstoneDJ, Swaminathan B, Eckstein J, Mundl H, Epstein AE et al (2019) Recurrent stroke with rivaroxaban compared with aspirin according to predictors of atrial fibrillation: secondary analysis of the NAVIGATE ESUS randomized clinical trial. JAMA Neurol 76(7):764-773

17. Ntaios G, Swaminathan B, Berkowitz SD, Gagliardi RJ, Lang W, Siegler JE et al (2019) Efficacy and safety of rivaroxaban versus aspirin in embolic stroke of undetermined source and carotid atherosclerosis. Stroke 50(9):2477-2485

18. Ntaios G, Pearce LA, Meseguer E, Endres M, Amarenco P, Ozturk S et al (2019) Aortic arch atherosclerosis in patients with embolic stroke of undetermined source: an exploratory analysis of the NAVIGATE ESUS trial. Stroke 50(11):3184-3190

19. Jalini $S$, Rajalingam $R$, Nisenbaum $R$, Javier $A D$, Woo A, Pikula A (2019) Atrial cardiopathy in patients with embolic strokes of unknown source and other stroke etiologies. Neurology 92(4):e288-e94

20. Tandon K, Tirschwell D, Longstreth WT Jr., Smith B, Akoum N (2019) Embolic stroke of undetermined source correlates to atrial fibrosis without atrial fibrillation. Neurology 93(4):e381-e7

21. Ramasamy S, Yaghi S, Salehi Omran S, Lerario MP Devereux R, Okin PM et al (2019) Association between left ventricular ejection fraction, wall motion abnormality, and embolic stroke of undetermined source. J Am Heart Assoc 8(9):e11593

22. Jordan K, Yaghi S, Poppas A, Chang AD, Mac Grory B, Cutting S et al (2019) Left atrial volume index is associated with cardioembolic stroke and atrial fibrillation detection after embolic stroke of undetermined source. Stroke 50(8):1997-2001

23. Eikelboom JW, Connolly SJ, Bosch J, Dagenais GR, Hart RG, Shestakovska O et al (2017) Rivaroxaban with or without aspirin in stable cardiovascular disease. NEngl J Med 377(14):1319-1330

24. Perera KS, Ng KKH, Nayar S, Catanese L, Dyal L, Sharma M et al (2019) Association between lowdose rivaroxaban with or without aspirin and ischemic stroke subtypes: a secondary analysis of the COMPASS trial. JAMA Neurol. https://doi.org/ 10.1001/jamaneurol.2019.2984

25. Geisler T, Poli S, Meisner C, Schreieck J, Zuern CS, Nagele T et al (2017) Apixaban for treatment of embolic stroke of undetermined source (ATTICUS randomized trial): rationale and study design. Int J Stroke 12(9):985-990

26. Kamel H, Longstreth WT Jr., Tirschwell DL, Kronmal RA, Broderick JP, Palesch YY et al (2019) The atrial cardiopathy and antithrombotic drugs in prevention after cryptogenic stroke randomized trial: rationale and methods. Int J Stroke 14(2):207-214. https://doi.org/10.1177/ 1747493018799981

27. Wachter R, Groschel K, Gelbrich G, Hamann GF, Kermer P, Liman J et al (2017) Holter-electrocardiogram-monitoring in patients with acute ischaemic stroke (Find-AFRANDOMISED): an openlabel randomised controlled trial. Lancet Neurol 16(4):282-290

28. Haeusler KG, Kirchhof P, Heuschmann PU, Laufs U, Busse O, KunzeCetal (2016) Impact of standardized MONitoring for detection of atrial fibrillation in ischemic stroke (monDAFIS): rationale and design of a prospective randomized multicenter study. Am Heart J 172:19-25

29. Diener HC, Deutsche Gesellschaft für Neurologie (DGN), Grau AJ, Deutsche Schlaganfall-Gesellschaft (DSG), Baldus S, Deutsche Gesellschaft für Kardiologie-, Herz- und Kreislaufforschung (DGK) et al (2018) Kryptogener Schlaganfall und offenes Formaen ovale: S2e Leitlinie [Cryptogenic stroke and patent foramen ovale: S2e guidelines]. Nervenarzt 89(10):1143-1153 